\title{
Beyond the Homestay Model: Peer Mentorship and Early Exposure in Global Health Education
}

\author{
Stefan Wheat ${ }^{1} \cdot$ Richard Mendez $^{1} \cdot$ Ruth Musselman $^{2} \cdot$ Fadzai Mugadza $^{3}$. \\ Samson Shumbairerwa $^{3}$ - Chiratidzo Ndhlovu ${ }^{4,3}$ • Patricia Wetherill ${ }^{2} \cdot$ Majid Sadigh $^{4}$. \\ Stephen Winter ${ }^{2}$
}

Published online: 27 April 2016

(C) International Association of Medical Science Educators 2016

\section{Introduction}

Participation and interest in global health electives has increased dramatically over the last decade across the spectrum of graduate health education, including trainees in medicine, public health, dentistry, and nursing [1-3]. This change reflects the increasingly global nature of healthcare delivery that is a consequence of the ease with which people and diseases may traverse borders. Global health training and participation in international electives have been recognized as a valuable addition to health education. Students participating in global health electives gain important insight into alternative models of healthcare delivery, develop an understanding of the challenges of health care delivery in resource constrained settings, have exposure to the ways in which culture may affect health care delivery, learn to trust clinical assessment skills in the absence of sophisticated imaging and monitoring systems, and see a spectrum of new and atypical disease presentations $[4,5]$. This may be coupled with the opportunity to learn important skills in carrying out research projects under the supervision of faculty. These experiences help to shape a more well-rounded healthcare professional and guide careers in

Stefan Wheat

Stefan.Wheat@med.uvm.edu

1 University of Vermont College of Medicine, Given 131, 89 Beaumont Avenue, Burlington, VT 05401, USA

2 Western Connecticut Health Network, 34 Maple Street, Norwalk, CT 06850, USA

3 University of Zimbabwe College of Health Sciences, 178 Avondale, Harare, Zimbabwe

4 Western Connecticut Health Network, 24 Hospital Avenue, Danbury, CT 06810, USA health toward a framework that appreciates the truly globalized nature of healthcare today [6].

Despite the clear advantages conferred by a global health elective and the growth of this aspect of health education, there remain numerous structural limitations for these programs, largely related to the passive approach of former models in which students are sent to observe the healthcare system of a developing country with little preparation and with no capacity to enhance or support that system. These limitations include a lack of bidirectional exchange between student country and host country, the tendency toward cultural isolation by global health elective students, and a shortage of psychosocial support for participating students [7, 8]. Additionally, the ad-hoc approach to global health education in which medical students participate in a global health elective at the end of their fourth year without substantial background training is unlikely to promote a lasting commitment toward future global health involvement, thereby suggesting the need for earlier exposure. These problems, which pervade previously practiced models of the global health elective, form barriers to the development of globally minded healthcare professionals, highlighting the need for new models that directly address the limitations while still encouraging the growth of global health electives and cross-cultural exchange.

Numerous models have been proposed to address the problems with former approaches to the global health elective, including the Homestay model presented by Chia et al [9]. This model, implemented in Kampala, Uganda, places a strong emphasis on cultural integration, emotional support and mentorship, and the development of cultural competency by placing visiting students in the household of a local faculty member to share meals and experiences with the host family. This is a powerful and effective model that requires a host family with the capacity to lodge multiple visiting students while instilling cultural competency, giving emotional support 
and providing mentorship, all in the language of the visiting students. This represents a significant logistical threshold and thus may not be widely generalizable.

This paper is meant as a follow-up to the Homestay model to describe another model that still addresses the challenges of the global health elective while having the added advantage of being applicable more broadly when the requirements of the Homestay model may not be achievable. We believe that there is tremendous value in the Homestay model which specifically addresses the problems of poor psychosocial support and cultural isolation that may be seen with previous models. However, identifying other suitable models for the global health elective is critical as the difficulties associated with establishing lasting and positive homestay relationships for students may be logistically prohibitive. The Peer Mentorship/Early Exposure model of the global health elective attempts to address the same issues addressed by the Homestay model, replacing the support and cultural integration provided by the host family with a team of host country medical students, residents, and attending physicians. Furthermore, the Peer Mentorship/Early Exposure model goes beyond these issues to address other challenges in global health electives.

\section{Challenges of International Health Electives}

The following issues, often encountered with former models of the global health elective, serve to impede the fulfillment of the potential of such experiences, thereby diminishing the value of the global health elective as a training tool:

1) Lack of bidirectional exchange: As described in the Homestay model paper, the present paradigm of the global health elective is to design electives in the global South to serve students of the global North. Students come to gain cultural experiences, see exotic diseases, and learn new skills. They rarely come with the experience to transfer knowledge or build capacity [10]. This one-way knowledge transfer is at the core of previously practiced models of global health education. While the gold standard of bidirectional exchange remains an exchange of personnel between countries, this approach may be augmented by a more complete exchange of ideas in the host country by the use of a team approach with more senior members who can play a role in knowledge exchange and capacity building.

2) Insufficient cultural integration: The experience of living and learning in a foreign country can be stressful and disorienting. This stress, combined with the novelty of the experience, can lead to culture shock which may, in turn, diminish the value students are able to gain from participating in an international elective. 11 The strain of participating in a global health elective can lead to students failing to explore the cultural underpinnings of healthcare through cultural integration, ultimately limiting the value of their experience overall. This problem is exacerbated by placement of students in locations lacking in support of more experienced peers or faculty.

3) Deficient psychosocial support: Students may be emotionally paralyzed by witnessing what seems to be unnecessary suffering and death due to resource limitations without an outlet to explore and reconcile their own feelings of emotional distress [12].

4) Delayed exposure: The current paradigm of the global health elective remains for students to participate in global health electives late in the course of their training. While this approach selects for a more mature, welltrained cohort of students who should be more capable of participating in a meaningful way during their elective, it often fails to engender a lasting commitment to global health by limiting opportunities for exposure to global health during medical school and post graduate training when it would be most effective.

\section{Philosophy}

The dramatic rise in global health elective participation is reflected in the concurrent increase in demand for medical school programs that can accommodate this interest. Indeed, applicants to medical school and residency programs increasingly factor the capacity of medical school programs to provide the resources to participate in international electives into their matriculation decisions [13]. Moreover, many students entering medical school maintain their belief in medicine as a vehicle for social change and the alleviation of suffering found in refugee, immigrant, and other marginalized populations. Thus, it is apparent that students, spurred by a genuine interest in issues of social justice, aspire to contribute to the burgeoning field of global health earlier than can typically be accommodated [14]. Moreover, students who do not enter medical school with a firm interest in global health are commonly swayed by the enthusiasm of their classmates [15]. By striving to bridge the gap between interest and capacity, we invest in future leaders and help guide the next generation of citizens of the world: individuals who will become instruments of social change, capable of addressing the increasing healthcare disparities around the world.

\section{The Peer Mentorship/Early Exposure Model}

The partnership between Western Connecticut Health Network (WCHN) and the University of Vermont College of 
Medicine (UVCOM) has led to a variety of global health opportunities for students at UVCOM with programs in Zimbabwe, Uganda, Vietnam, Russia and the Dominican Republic. This collaboration includes relationships with the University of Zimbabwe College of Health Sciences (UZCHS) and Makerere University College of Health Sciences (MUCHS). The MUCHS collaboration led to the development of the Homestay model of the international health elective which relies on relationships with physicians in Kampala, Uganda, willing to provide a host family experience for participating students. This model could not be easily generalized to other settings and we have developed an alternative model which we have applied at UZCHS. We believe that this alternative model accomplishes the same goals of cultural understanding, social support, and mutual exchange laid out in the Homestay model paper. The Peer Mentorship/ Early Exposure model begins with education of students during their first year in medical school and involves students participating in two international attachments, once in the summer after their first year and once during their fourth year. Students are first selected based on their interest in global health, their demonstrated ability to work in a demanding group context, and on their anticipated contribution to the field. Following selection, students prepare for their first international elective placement via pre-departure training (e.g. tropical diseases, ethics in global health, etc.). The model combines direct exposure to global health with a curriculum that spans the entirety of their education as medical students, including ongoing lecture material and the option to participate in globally minded clerkship electives (e.g., travel clinic, refugee population) throughout the remaining 3 years in medical school. This longitudinal education in global health culminates in students returning to the host-country they worked in after their first year during their fourth year. The aim of this paper is a description of the experience of the first group of elective students operating under the auspices of the Peer Mentorship/Early Exposure model.

\section{General Structure}

Our team was composed of two rising second year medical students, one senior internal medicine resident, and rotating attending physicians from the home country. The medical students and resident were on attachment in Harare for 6 weeks with attending physicians rotating every 2 weeks. The key element of this model is the close interaction between medical students and their resident, who serves as a peer mentor with close oversight by an experienced attending physician. Students participating in a global health elective at the University of Zimbabwe College of Health Sciences live in a flat within the medical student dormitories. The flat can accommodate up to four students. It is equipped with a kitchen to allow students to reduce the costs of living by cooking their own meals and has a living room with kitchen table to promote a community atmosphere among elective students and their resident physician mentor. In the first year of the program, two second year medical students lived with a resident physician in the flat while the attending physician lived offsite. The global health team-composed of two medical students, a resident, and an attending physician - attended morning rounds led by a Zimbabwean faculty member and the visiting attending and senior resident were included in the rounding teaching and patient management decision-making by providing input based upon their own specialty training and the perspectives and approach of western health care.

Elective student excerpt:

We spend the majority of our day alongside our resident, from enjoying oatmeal together in the morning to toiling over the endlessly frustrating library wifi in the late evening. The intimacy of this relationship, where medical student and resident mentor live and work together, forms the foundation that drives the success of this program. As the core of the global health team, we collaborate closely to design our day according to the preferences of each member of the group. When we need to take a step back and have a tutorial, we can. Similarly, if we need to take an afternoon off to catch our breath, the closeness of our relationship with our peer mentor allows us to feel comfortable doing so. Thus, the global health experience can be tailored to the dynamic educational and emotional needs of each participant.

\section{Bidirectional Exchange}

The team is explicitly designed to promote exchange of knowledge and ideas with the host physicians at every level. The entire team is linked to a ward team at Parirenyatwa Hospital, the primary teaching hospital for the medical school and post graduate training programs in the Department of Medicine at the University of Zimbabwe. This real time exchange of experience between the senior faculty and resident from our team and our host physicians breaks down cultural barriers and affects a capacity building role.

Host-country physician excerpt:

One of the visiting attending physicians requested to teach a procedure (i.e. lumbar puncture). We appreciated this because it allowed our own students to see an infectious disease specialist with extensive experience perform the procedure. This helped our own ward teams where the Junior Resident Medical Officer (JRMO) is left to do most of these procedures without real-time supervision. These junior practitioners are expected to know what to do once they have graduated but they 
often need additional assistance. Other members of the team contributed elsewhere as they had expertise in varying disciplines, including one attending physician who helped our bronchoscopy trainees. From my own point of view, having the resident and faculty members actually was a relief since we knew the visiting medical team would be self-sufficient, thereby reducing the burden that would have otherwise been placed on our own attending physicians to manage the educational needs of visiting students. I was amazed at how complimentary the visiting team was in our ward rounds.

This is augmented by a similar process after ward rounds and on admitting nights in the emergency department where our senior resident, along with the students, would provide a similar and complementary exchange of ideas at the bedside with their resident level peers in training. The attending physician was also expected to provide formal didactic lectures and workshops each afternoon for the Zimbabwean trainees. Thus, a nearly continuous process of exchange and capacity building was fully integrated at both a peer and faculty level. Another advantage of the team approach to the global health elective is how this model can serve as an example of crossnational collaboration, thereby empowering host-country students.

Host-country physician excerpt:

Due to the differences in medical practice between Zimbabwe and more developed countries, it remains difficult for many of our medical students and residents to fully appreciate that they can share clinical experience and evidence-based medical practices with their first world counterparts. Hosting a Critical Care specialist from the US was a blessing that meant that the host consultant and the attending faculty member interacted during clinical rounds in the ICU, both using bedside teaching methods in which an emphasis on basic sciences was demonstrated and our residents appreciated it as a common denominator in Critical Care. This also allowed the host consultant and the visiting attending faculty member to interact and provide an example of cross-cultural cooperation for Zimbabwean students. Afternoon interactive tutorials were conducted daily and among the most exciting was the question and answer session where both the host and attending faculty were there to respond to the residents' questions which ranged from ethics to more discrete clinical scenarios related to Critical Care and End of life issues. This model of global health education conferred invaluable confidence on host-country residents as they realized that differences in resources among countries need not be an impediment to sharing evidence based medical knowledge/practices. The thrust therefore should be aimed at spreading the initiative of cross-country collaboration and exchange across all medical specialties so that more aspects of global health education can benefit from the advantages it brings.

\section{Cultural Integration}

Elective students are made privy to the cultural nuances of the host country in several ways. First, students live within the host-country student dormitories. This intimate living experience with host-country peers combined with the fact that elective students round and attend clinic with Zimbabwean medical students provides the elective students with extensive opportunities for close interaction with local students, thus facilitating meaningful exchange. Second, an important component of cultural understanding in a medical setting is recognizing the differences between origin country and host country practices. These differences may not be clear to students in their pre-clinical years. Thus, the team structure adds this important insight to the understanding of cultural differences that is ultimately valuable for both elective students and their hostcountry counterparts.

Resident physician excerpt:

The family approach to death was also a unique teaching point when it came to contrasting cultural nuances. Having worked as a resident for two years in the US health care system I have been accustomed to the concept of family being present for the end of life and typically playing an active role in end of life decision making. In contrast, the vast majority of patients dying in this hospital are alone in the hospital bed. Visiting hours are strict, so even if death is imminent, families will typically not even hear of the death of a loved one until the next hour allotment. As a team we discussed the contrast to the dying process at home: in the US, family members are encouraged to stay at the bedside and are typically present at the time of dying. As a doctor we are also expected to guide patients and family through the process at every turn. The same level of attention at the end of life is not the standard here, and discussing this difference emerged as an important point of education for the elective students.

Host-country resident physician excerpt:

Despite the differences related to resource limitations, the contribution of the visiting team highlighted the parallels that could be drawn in our conduct and practice in medicine. In the ICU, end of life care emerged as a topic of particular interest. We shared our beliefs and cultural values pertaining to end of life care and how these values inform medical practice. It was interesting to hear 
from the visiting team and compare how their values intersected and diverged from our own. Interestingly, although there were clear contrasts between the value structures underpinning end of life care decisions in Zimbabwe and the US, parallels could be drawn in certain aspects of how we approached this most delicate issue. Equipped with insight into these two practices, I approached terminally-ill patients and their family with a new perspective.

Therefore, the presence of a peer mentor resident in addition to the close support provided by the attending physician confers a more concrete understanding of these cultural and medical system differences, allowing for a more nuanced appreciation of the host-country's healthcare system.

\section{Psychosocial Support}

The close relationship between resident, attending faculty, and medical students - which is the aim of the Peer Mentorship/Early Exposure model —overtly establishes a framework that promotes a sense of community and a group dynamic where participants can freely voice their frustrations. Most patients presenting to Parirenyatwa Hospital come with advanced disease and mortality rates are high, presenting a degree of human suffering that may be new for resident and attending physicians as well as their students. The team makeup, including an experienced senior resident and an attending with prior experience in global health provided an outlet for emotional support at a peer level at the bedside of sick patients, during late afternoon tutorials and discussion sessions with the attending, and on many nights over shared dinners.

\section{Elective student excerpt:}

In a resource limited setting, one quickly becomes acquainted with watching patients succumb to their illnesses. Combine this singularly visceral experience with a new and often disorienting environment and you wind up with a recipe for a potentially negative introduction to global health. In traveling and working with a familiar medical team, we are afforded an outlet to deal with the frequently challenging reality of healthcare delivery in Zimbabwe. The inability to help as you stand at the bedside of patients in respiratory arrest is frustrating, and the medical team relationships proved to be an essential component of coping with these difficult daily experiences. Rather than wearing me down to the point of exhausted despondence, the frustrations of working in this trying environment instead forged a strong bond amongst members of the medical team.
Participants navigate the challenges of working in a global health setting together and, through this shared experience, are able to serve as a support-network for one another.

\section{Early Exposure}

While it is well established that students who participate in international electives during medical school or residency are more likely to tailor their careers to work with underserved and immigrant patient populations at home, it is not clear that global health electives effectively engender a sustained commitment to working in an international setting [16-18]. Though some evidence exists to suggest that students who participate in international electives are more likely to plan on working oversees in the future, this evidence is limited to resident physician populations [19]. Earlier experiences have the potential to more profoundly influence decisions that shape careers in medicine [20]. Indeed, a lack of commitment to working internationally may be a consequence of not integrating students into the discussion surrounding the expanding field of global health early enough [21]. In order to breed lasting commitment and interest in the issues of global health, elective students should be made to feel as active participants in the field, rather than passive bystanders, a characteristic of previously practiced models of global health. To address this problem, our model offers the solution of early exposure with follow-up. By introducing students to a global health setting early in their education, supplementing this experience with ongoing education in subjects pertaining to global health throughout all 4 years of medical school and culminating in students returning to the same host country at the end of medical school, elective participants are more deeply integrated into the global health conversation. Highlighted by two international attachments - one after the first year of medical school and again during the fourth year-medical students participating in the Peer Mentorship/Early Exposure model engage in global health education throughout the course of medical school, including electives working with refugee populations and working in a travel clinic.

The value added by early exposure is bolstered by the advantages of the global health team and Peer Mentorship/Early Exposure model, a model that further addresses the problem of commitment to issues in global health in elective students. Gaining a nuanced comparative perspective of two disparate healthcare delivery systems allows students to become participants in the dialog regarding the evolving model of global health. This is made possible by the Peer Mentorship/Early Exposure model insofar as students are privy to conversations with host country physicians regarding the state of medicine in their country. These conversations are facilitated by having home country resident and attending physicians who can offer engaging discussion on matters that would typically be beyond the scope of learning for first year medical students. 
The depth of this interaction provides for a more culturally enriching experience for students.

Elective student excerpt:

We were recently invited by one of the senior consultants at the hospital to a traditional Zimbabwean meal of sadza, beef stew, collard greens, and peanut-butter rice (my personal favorite). The ensuing conversation represents what I think was one of the most significant encounters of our time in Zimbabwe. We were given a glimpse into the grim history of how the healthcare industry has changed in Zimbabwe over the course of the last decade. Indeed, learning how the debilitating inflation in Zimbabwe changed the face of medicine, and particularly medical education, helped to place in context some of the incongruous behavior we witnessed working on the wards. The delicious meal and impassioned account of the history of medicine in Zimbabwe we received that night seemed to be a gesture of gratitude toward our attending physicians for their teaching. I was simply the fortunate tagalong.

The benefits of the mentorship between elective participants and their resident and attending physicians extend beyond the international setting, with students encouraged to engage in scholarly pursuits upon their return to the USA, including attending global health conferences and seeking publication. This early exposure to a comparison between two medical systems along with their respective cultural and historical underpinnings, combined with the continuing education in global health throughout medical school engenders a more lasting appreciation of the value added by working in a global health setting. Moreover, with direct access to resident and attending physicians who are deeply invested in global health, students are afforded the opportunity to develop a clear path toward future involvement under the mentorship of senior faculty.

\section{Limitations of the Model}

1. The Peer Mentorship/Early Exposure model does not include a built-in and assured link to the local community as the Homestay model does. Our model relies on the maturity of elective students and their peer mentors to recognize and seek out the cultural integration that will serve to enrich their experience. Therefore, it is important that the chosen participants be suitably able to explore outside their realm of comfort. An international elective curriculum that includes training in cultural sensitivity, language, and host-country sociopolitical context could also serve to ameliorate this limitation.

2. The support network that forms in the Peer Mentorship/ Early Exposure model is dependent on selecting a group who are adaptable and mutually supporting. This is made possible by giving global health elective students sufficient time to identify counterparts who they can work and live with. Attempting to form groups who will develop positive relationships among themselves and with their resident peer mentor is crucial to the success of this model, and - as with the Homestay model - requires a careful selection process and orientation of all involved [22].

3. The demand for attending resources in this model is high. We have chosen to provide faculty mentorship and supervision for the entire 6-week rotation in three 2-week rotations. In our other global health elective settings, we have included senior subspecialty fellows as faculty to supplement more senior faculty with excellent results. We have also considered limiting faculty level involvement to only the first 23 weeks of the elective for future groups to address this problem. Though full faculty coverage of the elective should be sought after as the gold standard, we believe that an approach with limited faculty coverage could achieve similar results to coverage of the full rotation by establishing an early foundation of partnership between the visiting and local teams.

4) Many locations for training in developing countries do not have the capacity or adaptability to easily incorporate a foreign team. We were fortunate to be at a hospital and medical school that was accustomed to foreign medical teams. The Department of Medicine's guidance allowed us to quickly assimilate and understand our role in the day to day workings of the ward. The ease of this transition was facilitated by the efforts of the attending physicians who served as the primary advocates of the team.

\section{Conclusion}

The increasingly globalized nature of healthcare underlies the importance of education of health professionals in a global context. With the recent explosion of participation in global health electives across health disciplines, it is imperative that we consider how to maximize the value added for elective students but also for the healthcare community at large. The enduring problems of bidirectional exchange, cultural immersion, psychosocial support, and durable commitment introduce challenges that may diminish the value of an international elective for students and potentially hinder the broader development of international relationships in the healthcare sector.

While the Homestay model represents one approach to promoting cultural immersion and understanding, the development of such unique relationships with host families can introduce additional challenges. The Peer Mentorship/Early Exposure model presented above is an alternative model that may serve to limit these additional challenges while still accomplishing the goals of cultural understanding and 
psychosocial support. Through the shared experience of working and living together in an international setting, the core medical team is emboldened to seek culturally enriching experiences while retaining the safety-net and emotional foundation of the group. Explicitly designed to support the educational and emotional needs of the elective students, the team approach intrinsic to our model similarly provides capacity building support to the host-country medical system via formal instruction while simultaneously limiting the burden placed on host-country physicians to oversee the instruction of visiting students. Via this team approach, resident and attending physicians serve as mentors to elective students, developing a pedagogical relationship that persists well after the international elective concludes. This structure, coupled with the model of ongoing education in global health throughout elective students' education, engenders a culture of mutual appreciation between host country practitioners and the visiting medical team and imparts a degree of appreciation for the value of working in an international setting that may be lost on students engaging in isolated electives near the end of their education. The Peer Mentorship/Early Exposure model thus allows participating students to cultivate a deeper commitment to working internationally.

Acknowledgments We wish to thank Dr. Andrew Mataruse, Dr. Sara Lowe, Dr. Margaret Borok, Dr. Andrew Reid, Dr. Gift Ngwende, and Dr. Pamela Gorejena for their support in Zimbabwe. We would also like to thank the University of Zimbabwe College of Health Sciences, Western Connecticut Health Network, and the University of Vermont College of Medicine.

\section{Compliance with Ethical Standards}

Funding This study was funded by the Western Connecticut Health Network Global Health Program.

Conflict of Interest The authors declare that they have no competing interests.

\section{References}

1. Izadnegahdar R, Correia S, Ohata B, et al. Global health in Canadian medical education: current practices and opportunities. Acad Med. 2008;83(2):192-8.

2. Rowson M, Smith A, Hughes R, et al. The evolution of global health teaching in undergraduate medical curricula. Global Health. 2012;8:35.

3. Amaya-Burns A, Fesperman S, Non A, Amaya M, Evans K. Establishing a sustainable partnership through short-term global field experiences: the University of Florida and Canton el Limón collaboration. Public Health Reports. 2010;125(3):500-4.

4. Sawatsky AP, Rosenman DJ, Merry SP, McDonald FS. Eight years of the Mayo International Health Program: what an international elective adds to resident education. Mayo Clin Proc. 2010;85(8): 734-41.

5. Henry JA, Groen RS, Price RR, et al. The benefits of international rotations to resource-limited settings for U.S. surgery residents. Surgery. 2013;153(4):445-54.

6. Gupta AR, Wells CK, Horwitz RI, Bia FJ, Barry M. The International Health Program: the fifteen-year experience with Yale University's internal medicine residency program. AmJTrop Med Hyg. 1999;61(6):1019-23.

7. Battat R, Seidman G, Chadi N, et al. Global health competencies and approaches in medical education: a literature review. BMC Med Educ. 2010;10:94.

8. Eichbaum Q. The problem with competencies in global health education. Acad Med. 2015;90(4):414-7.

9. Chia D, Sadigh M, Goller T, et al. A homestay model for global health and medical education in resource-limited settings. Med Sci Educ. 2015;25(3):317-21.

10. Kumwenda B, Dowell J, Daniels K, Merrylees N. Medical electives in sub-Saharan Africa: a host perspective. Med Educ. 2015;49(6): 623-33.

11. Lewthwaite M. A study of international students' perspectives on cross-cultural adaptation. Int J Adv Counselling. 1996;19(2):16785 .

12. Wear D. "Face-to-face with it": medical students' narratives about their end-of-life education. Acad Med. 2002;77(4):271-7.

13. Dey CC, Grabowski JG, Gebreyes K, Hsu E, VanRooyen MJ. Influence of international emergency medicine opportunities on residency program selection. Acad Emerg Med. 2002;9(7):679-83.

14. Peluso MJ, Forrestel AK, Hafler JP, Rohrbaugh RM. Structured global health programs in U.S. medical schools: a web-based review of certificates, tracks, and concentrations. Acad Med. 2013;88(1):124-30.

15. Huggett K. An introduction to medical teaching. New York: Springer; 2014.

16. Godkin M, Savageau J. The effect of medical students' international experiences on attitudes toward serving underserved multicultural populations. Fam Med. 2003;35(4):273-8.

17. Thompson MJ, Huntington MK, Hunt DD, Pinsky LE, Brodie JJ. Educational effects of international health electives on U.S. and Canadian medical students and residents: a literature review. Acad Med. 2003;78(3):342-7.

18. Ramsey AH, Haq C, Gjerde CL, Rothenberg D. Career influence of an international health experience during medical school. Fam Med. 2004;36(6):412-6.

19. Miller WC, Corey GR, Lallinger GJ, Durack DT. International health and internal medicine residency training: the Duke University experience. Am J Med. 1995;99(3):291-7.

20. Drain PK, Primack A, Hunt DD, Fawzi WW, Holmes KK, Gardner P. Global health in medical education: a call for more training and opportunities. Acad Med. 2007;82(3):226-30.

21. Ledley FD, Lovejoy Jr FH. Factors influencing the interests, career paths, and research activities of recent graduates from an academic, pediatric residency program. Pediatrics. 1993;92(3):436-41.

22. Torjesen K, Mandalakas A, Kahn R, Duncan B. International child health electives for pediatric residents. Arch Pediatr Adolesc Med. 1999;153(12):1297-302. 